\title{
Effects of preset sequential administrations of sunitinib and everolimus on tumour differentiation in Caki-1 renal cell carcinoma
}

\author{
C Dos Santos ${ }^{1,2}$, A Tijeras-Raballand ${ }^{1,2}$, M Serova ${ }^{1,2}$, S Sebbagh ${ }^{2}$, K Slimane ${ }^{3}$, S Faivre ${ }^{4}$, A de Gramont ${ }^{1,4,5}$ \\ and E Raymond*,2,4,5 \\ ${ }^{1}$ AAREC Filia Research, 1 place Paul Verlaine, 92100 Boulogne Billancourt, France; ${ }^{2}$ Inserm U728, Department of Medical Oncology, \\ Beaujon University Hospital, 100 Boulevard du general Leclerc, 92110 Clichy, France; ${ }^{3}$ Novartis Pharma SAS, 2 et 4 rue Lionel Terray, \\ 92506 Rueil Malmaison, France and ${ }^{4}$ New drugs Evaluation Laboratory, Center of Experimental Therapeutics and Medical Oncology, \\ Oncology Department, CHUV, Lausanne Switzerland
}

Background: Sunitinib (VEGFR/PDGFR inhibitor) and everolimus (mTOR inhibitor) are both approved for advanced renal cell carcinoma (RCC) as first-line and second-line therapy, respectively. In the clinics, sunitinib treatment is limited by the emergence of acquired resistance, leading to a switch to second-line treatment at progression, often based on everolimus. No data have been yet generated on programmed alternating sequential strategies combining alternative use of sunitinib and everolimus before progression. Such strategy is expected to delay the emergence of acquired resistance and improve tumour control. The aim of our study was to assess the changes in tumours induced by three different sequences administration of sunitinib and everolimus.

Methods: In human Caki-1 RCC xenograft model, sunitinib was alternated with everolimus every week, every 2 weeks, or every 3 weeks. Effects on necrosis, hypoxia, angiogenesis, and EMT status were assessed by immunohisochemistry and immunofluorescence.

Results: Sunitinib and everolimus programmed sequential regimens before progression yielded longer median time to tumour progression than sunitinib and everolimus monotherapies. In each group of treatment, tumour growth control was associated with inhibition of mTOR pathway and changes from a mesenchymal towards an epithelial phenotype, with a decrease in vimentin and an increase in E-cadherin expression. The sequential combinations of these two agents in a RCC mouse clinical trial induced antiangiogenic effects, leading to tumour necrosis.

Conclusions: In summary, our study showed that alternate sequence of sunitinib and everolimus mitigated the development of mesenchymal phenotype compared with sunitinib as single agent.

The incidence of renal cell carcinoma (RCC) has been rising steadily and now accounts for $2-3 \%$ of all adult cancers worldwide (Cohen and McGovern, 2005; Ljungberg et al, 2013). Clear cell carcinoma (ccRCC) stands as the main pathological subtype (80-90\%), papillary RCC and chromophobe RCC being the two other major types of RCC according to the WHO tumour classification. Recent data from The Cancer Genome Atlas (TCGA) identified ccRCC as a highly metabolically deregulated cancer and a model for the role of a metabolic shift or 'Warburg effect' observed in the acquisition of cell malignancy. Molecular characterisation of ccRCC and preclinical investigations highlighted the importance of the von Hippel-Lindau (VHL) gene alterations (Creighton et al, 2013), inhibition of VHL function through mutations, or epigenetic silencing occurring $\sim 60-75 \%$ of

\footnotetext{
${ }^{*}$ Correspondence: Professor E Raymond; E-mail: eric.raymond@bjn.aphp.fr
}

${ }^{5}$ These authors equally contributed to this work. 
tumours (Gossage et al, 2014). von Hippel-Lindau is an E3 ubiquitin ligase that targets the hypoxia-inducible transcription factors (HIFs), HIF1 $\alpha$ and HIF2 $\alpha$, for degradation (Cockman et al, 2000). von Hippel-Lindau loss-of-function induces HIF1 $\alpha$ stabilisation and the constitutive transcription of several genes involved in differentiation, angiogenesis, and metabolism regulation (Pugh and Ratcliffe, 2003; Chiatar et al, 2013; Semenza, 2013). Integrated analyses from the TCGA also highlighted that other pathways, especially the chromatin remodelling and PI3K/AKT/mTOR pathways, were important in ccRCC tumourigenesis (Creighton et al, 2013). Interestingly, alterations in the PI3K/AKT/mTOR pathway can be identified in about $28 \%$ of tumours and are only partially overlapping with VHL dysfunctions. Consistent with metabolic dysfunctions in ccRCC, downregulation of the AMP-activated kinase, upregulation of pentose phosphate and fatty acid synthesis pathway genes, as well as upregulation of the $\mathrm{PI} 3 \mathrm{~K} / \mathrm{AKT} / \mathrm{mTOR}$ pathway correlated with worse survival (Creighton et al, 2013).

As a result of HIF activation by hypoxia or VHL mutations, as well as mTOR pathway dysfunctions, ccRCCs also acknowledged as highly vascularised, mainly through the paracrine secretion of vascular endothelial growth factors (VEGFs) by cancer cells. Inhibition of the VEGF receptors (VEGFRs) by small tyrosine kinase inhibitors (TKIs), such as sunitinib that was the first TKI approved for advanced RCC, markedly improved the progressionfree survival (PFS) and the overall clinical outcome of these patients (Schoffski et al, 2006; Faivre et al, 2006a). Sunitinib has a long stand as the standard of care for first-line therapy (Motzer et al, 2007) but despite evident clinical benefits, it is now accepted that most patients eventually progress after few months of therapy (Faivre et al, 2007). Loss of sunitinib activity was thought to be due to tumour cell plasticity that allows tumour cells, endothelial cells, and pericytes to adapt to their changing VEGFR-independent microenvironment by activating other survival and angiogenic pathways (Gassenmaier et al, 2013; Huang et al, 2013; Lu et al, 2013; Zhang et al, 2013). Data have also suggested that tumour adaptation to angiogenesis inhibition may trigger tumour aggressiveness, facilitating occurrence of metastasis (Ebos et al, 2009; Paez-Ribes et al, 2009). In renal cancer models, data have suggested that the $\mathrm{PI} 3 \mathrm{~K} / \mathrm{AKT} / \mathrm{mTOR}$ pathway could be involved in resistance to sunitinib (Makhov et al, 2012). Currently, clinical evidences of resistance to sunitinib lead most patients to switch for a secondline therapy based on either mTOR inhibitors (Faivre et al, 2006b) such as everolimus (Motzer et al, 2008, 2010) or on small TKIs whose inhibitory spectrum differs to some extend from sunitinib, such as sorafenib or pazopanib (Keisner and Shah, 2011; Afonso et al, 2013). In fact, emerging evidence showed that everolimus may display both antiangiogenic and antitumour activities and that these effects may counteract sunitinib resistance (Lane et al, 2009; Fuereder et al, 2010). Taking advantage of potentially distinct mechanism of action on cell signalling, authors have attempted to combine everolimus with sunitinib (Fuereder et al, 2010). However, these attempts were limited by significant, that is, severe dose-limiting acute and chronic toxicities (Molina et al, 2012).

Concomitant use of sunitinib and everolimus being prohibited by toxicity, sequential administration of both drugs has been proposed with the goal of switching drugs at fixed time points before acquired resistance may have a chance to emerge. In this study, we designed a preset strategy using sequential administration of sunitinib and everolimus before progression in human RCC xenograft models.

\section{MATERIALS AND METHODS}

Drugs. Everolimus was supplied by Novartis (Novartis Pharma SAS, Reuil-Malmaison, France). Sunitinib was purchased from LC Laboratories (Woburn, MA, USA). Sunitinib powder was dissolved immediately before gavage in a sterilised solution containing $0.5 \%$ carboxymethylcellulose, $0.4 \%$ Tween $80,1.8 \% \mathrm{NaCl}$ and $0.9 \%$ benzyl alcohol. Everolimus as a microemulsion was suspended in sterile water at an appropriate concentration and was administrated within $2 \mathrm{~h}$.

Cell line. Caki-1 ccRCC cell line was obtained from the ATCC (Rockville, MD, USA). The Cells were grown as monolayers in RPMI medium supplemented with $10 \%$ foetal calf serum, $2 \mathrm{~mm}$ glutamine, 100 units per $\mathrm{ml}$ penicillin and $100 \mu \mathrm{g} \mathrm{ml}^{-1}$ streptomycin (Invitrogen, Cergy-Pontoise, France), at $37^{\circ} \mathrm{C}$ in a humidified $5 \% \mathrm{CO}_{2}$ atmosphere, and regularly checked for absence of mycoplasma.

Tumour cells xenografts in nude mice. All the in vivo experiments were carried out with ethical committee approval and met the standards required by the UKCCCR guidelines. A total of $5 \times 10^{6}$ Caki-1 renal cancer cells were injected subcutaneously into the flank of female athymic nude mice (Janvier, Le Genest St Isle, France). One week after cell inoculation, all mice developed single subcutaneous palpable tumours of $\sim 50-100 \mathrm{~mm}^{3}$. Mice were arbitrarily placed in the groups of treatment. Mice were then treated 5 days a week by oral gavages with $60 \mathrm{mg} \mathrm{kg}^{-1}$ per day sunitinib $(n=5), 10 \mathrm{mg} \mathrm{kg}^{-1}$ per day everolimus $(n=5)$, or sequential regimens that alternate sunitinib and everolimus for 1 $(n=5), 2(n=5)$, or 3 weeks $(n=5)$, or only with the sterilised solution (control group, $n=6$ ). To choose the everolimus concentration, a pilot study was performed in this model using five different concentrations as following: 1, 2.5, 5, 10, and $20 \mathrm{mg} \mathrm{kg}^{-1}$ per day. Tumour growth inhibition was observed from $5 \mathrm{mg} \mathrm{kg}^{-1}$ per day, and was significant at 10 and $20 \mathrm{mg} \mathrm{kg}^{-1}$ per day. To minimise the toxicity in the sequential regimens, we choose the lowest effective concentration of everolimus, that is, $10 \mathrm{mg} \mathrm{kg}^{-1}$ per day. Moreover, this concentration was shown to delay tumour growth in vivo (O'Reilly et al, 2006; Larkin et al, 2012).Tumour volumes were measured twice a week along two major axes using a caliper. Tumour volumes were calculated as follows: tumour volume $=\left((\right.$ length $) \times\left(\right.$ width $\left.\left.^{2}\right)\right) / 2$. Tumours were considered progressive when, among the three consecutive increasing measures, tumour volumes displayed a 30\% increase compared with the smallest tumour measurement (NADIR). Time to tumour progression (TTP) was defined as the time from start of drug administration to first evidence of tumour progression. Our study defined two main tumour responses as following: (i) sustained (ST) tumour control, that is, partial response followed by tumour volume stabilisation or slow tumour volume progression, or (ii) tumour progression (PG), that is, tumour volume stabilisation followed by quick disease progression. Partial response in the treated groups was defined as a minimum of $30 \%$ tumour volume reduction from baseline. At day 66, when all placebotreated mice were killed, ST tumour control was confirmed whether tumour volumes had not doubled in size from baseline. If tumour volumes were over twice the baseline volumes, mice were considered to belong to the tumour PG group. When tumour volumes reached $2 \mathrm{~cm}^{3}$, mice were killed. Body and tumour weight were recorded. After mice were killed, tumours were excised and OCT embedded (VWR, Fontenay-sous-bois, France) to prevent tissue degradation from frozen conservation.

Immunohistochemistry analysis. The immunohistochemical procedure was performed on OCT-embedded subcutaneous tumour stained with haematoxylin and eosin (HE), and carbonic anhydrase 9 (CA-IX) (NB-100-417, Novus Biologicals, Littleton, CO, USA) using an automated immunohistochemical stainer. The images were captured and analysed with a Zeiss Observer Z1 microscope (Zeiss, Oberkochen, Germany). Quantifications were performed using Image J software (NIH, Bethesda, MD, USA) for HE and CA-IX staining. Necrotic areas were defined as tumour section 
areas where cells displayed pycnotic nucleus or no nucleus. Necrotic tissues are characterised by a strong pink colour, as observed in Figure 3.

Immunofluorescence analysis. Tumour slices were fixed in $4 \%$ paraformaldehyde (Invitrogen, Cergy-Pontoise, France), washed with PBS, where after unspecific sites were blocked using PBS containing $10 \mathrm{mg} \mathrm{ml}^{-1} \mathrm{BSA}$ for $1 \mathrm{~h}$ at room temperature (RT). Slices were then incubated with rat anti-human CD31 antibodies (550174BD Biosciences, San Jose, CA, USA), rabbit anti-human phospho-S6 ribosomal protein (pS6) (1/100), vimentin (1/100), or E-cadherin (1/100) primary antibodies (Cell Signaling, St Quentin en Yvelines, France) at RT for $1 \mathrm{~h}$, followed by incubation with the secondary antibodies Alexa Fluor (1/200) (Molecular Probes, Life Technologies, Saint-Aubin, France) for $1 \mathrm{~h}$ at RT and in the dark. Nuclei were stained with $5 \mu \mathrm{g} \mathrm{ml}^{-1}$ DAPI. Image analysis was performed using Zeiss Observer Z1 fluorescent microscope (Zeiss, Oberkochen, Germany) and Image J software.

Statistical analysis. The significance of variability between the results of each group and its corresponding control was determined by unpaired $t$-test using GraphPad Prism 5 (GraphPad Software Inc., San Diego, CA, USA). For immunohistochemistry and immunofluorescence analysis, values represent the means \pm s.e.m. of at least five quantifications per animal. Survival curves were plotted according to Kaplan-Meier.

\section{RESULTS}

Effects of sequential administrations of sunitinib and everolimus on tumour PG. Caki-1 cells were subcutaneously injected into nude mice. After 1 week, when tumours became palpable $\left(50-100 \mathrm{~mm}^{3}\right)$, the mice were randomly assigned to six treatment groups including vehicle control, sunitinib $60 \mathrm{mg} \mathrm{kg}^{-1}$ per day, everolimus $10 \mathrm{mg} \mathrm{kg}^{-1}$ per day, or alternate administration of sunitinib (S) and everolimus (E) every week (1W), every 2 weeks $(2 \mathrm{~W})$, or every 3 weeks ( $3 \mathrm{~W}$ ) (Figure 1A). Tumour growth was followed by measuring tumour volume twice a week (Supplementary Figure 1). Tumours were considered progressive when, among three consecutive increasing measures, tumour volumes displayed $a \geqslant 30 \%$ increase compared with the smallest tumour measurement. Median TTP (mTTP) in vehicle, sunitinib, and everolimus groups was 24, 36, and 50 days, respectively (Figure 1B). When analysed as a single group, alternated sequential treatments improved the mTTP to 59 days. More specifically, mTTP in the 1,2 , and $3 \mathrm{~W}$ groups were 64, 59, and 59 days, respectively (Figure 1C). This improvement represented a 2.5 -fold increase in mTTP compared with the control treatment group and a $20-80 \%$ increase compared with single agents sunitinib or everolimus.

Effects of preset sequences of sunitinib and everolimus on tumour angiogenesis. Tumour angiogenesis was analysed by CD31 staining, a surface marker of endothelial cells allowing evaluation of the number of vessel and the vascular area of tumours (Figure 2A). In all treated groups, the number of vessels was significantly decreased relative to the control group (Figure 2B). In accordance with the known antiangiogenic effects of sunitinib, sunitinib alone and alternated sequences containing sunitinib induced a reduction in the number of vessels in comparison to treatment with everolimus alone. In addition, the vascular areas were significantly decreased in all of the treated tumours compared with control, confirming that sunitinib- and
A

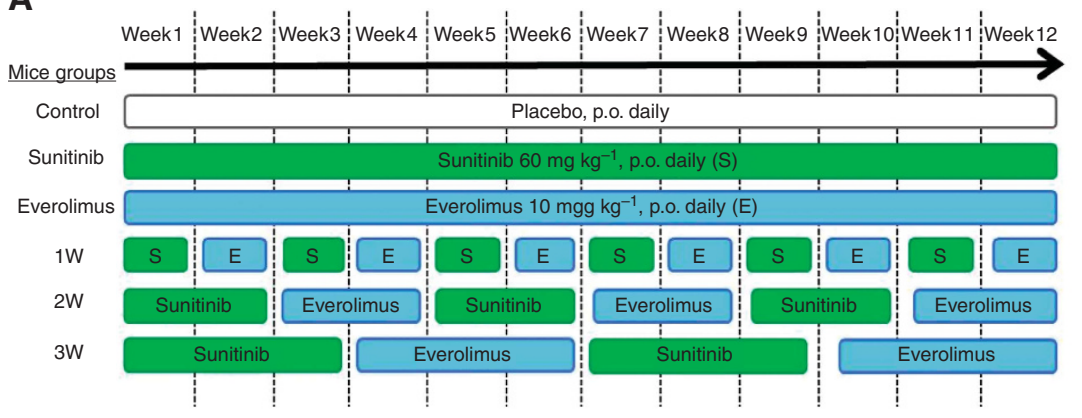

B

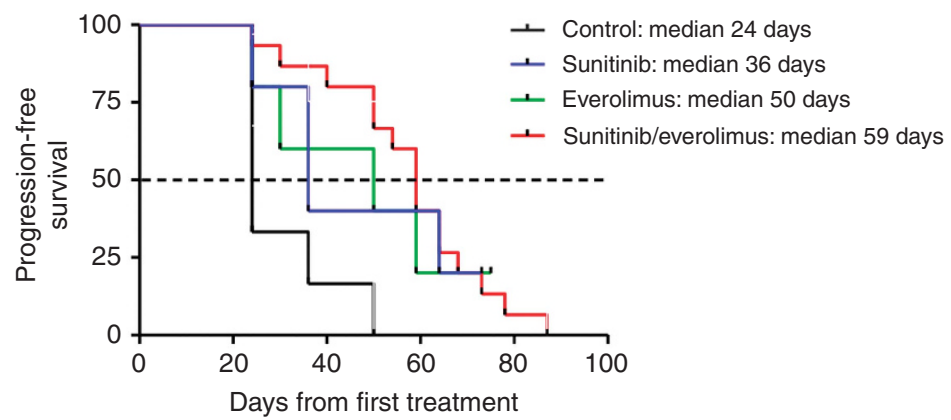

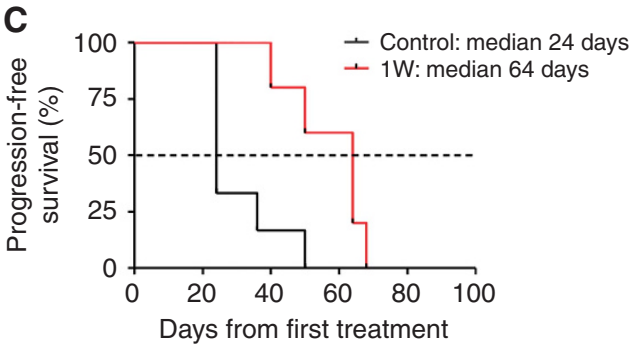
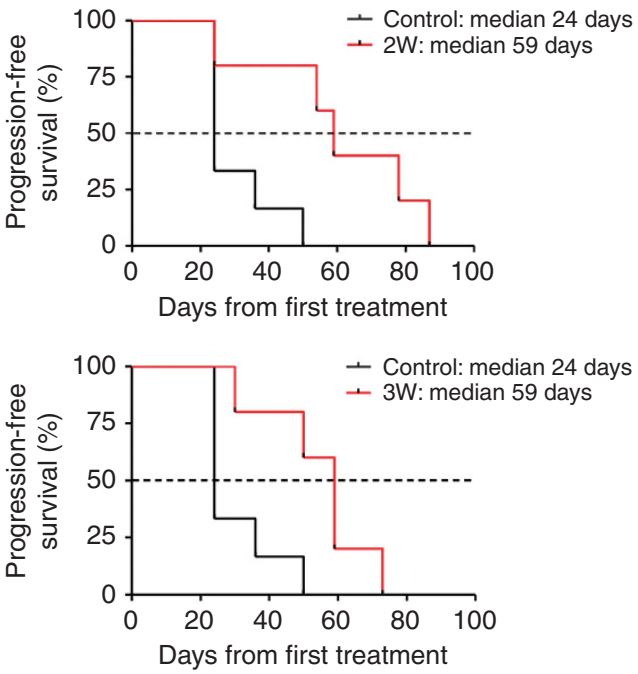

Figure 1. TTP of RCC xenografts treated with vehicle, sunitinib, everolimus, and sequences. Mice groups according to the treatment protocols (A) Kaplan-Meier evaluations for mice treated with $60 \mathrm{mg} \mathrm{kg}^{-1}$ per day sunitinib, $10 \mathrm{mg} \mathrm{kg}^{-1}$ per day everolimus, sequential combination of $60 \mathrm{mg} \mathrm{kg}^{-1}$ per day sunitinib, and $10 \mathrm{mg} \mathrm{kg}^{-1}$ per day everolimus as a single group (B) composed with treatment of alternate drugs administration every week (1W), every 2 weeks (2W), and every 3 weeks $(3 W)(C)$. 
A
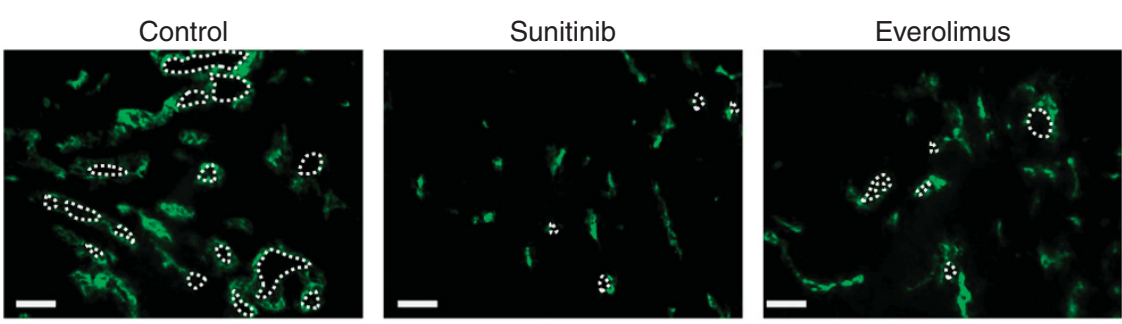

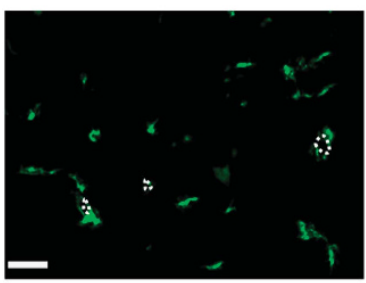

$1 \mathrm{~W}$

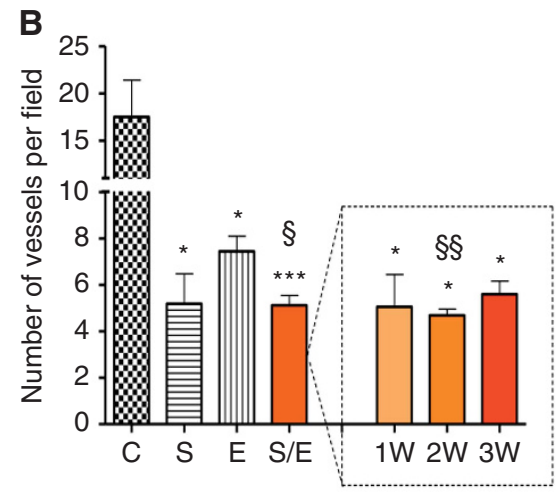

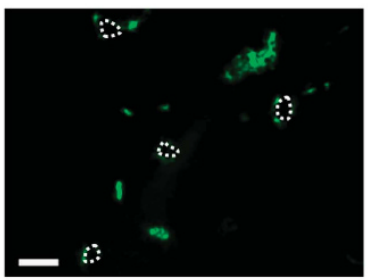

$2 \mathrm{~W}$

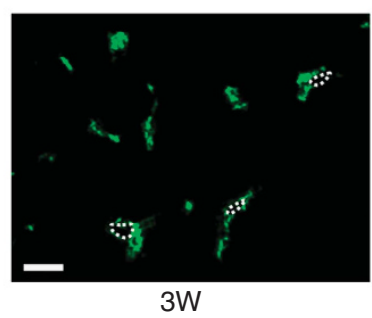

C

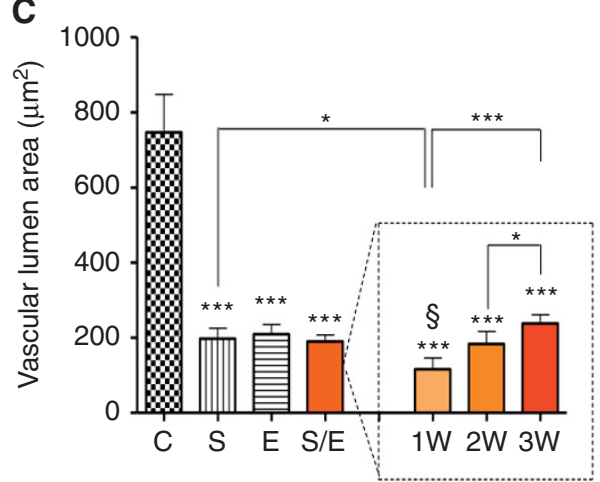

Figure 2. Immunofluorescence analysis of angiogenesis from Caki-1 tumour sections stained with CD31. Representative pictures of CD31 staining for each group of treatment. Bar graph $=200 \mu \mathrm{M}(\mathrm{A})$. Angiogenesis was quantified using Axiovision software (Zeiss, Oberkochen, Germany); values represent the means \pm s.e.m. of at least five quantifications per animal. Number of vessels (B) and vascular lumen area (C) per

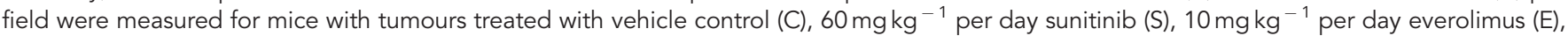
sequential combination of $60 \mathrm{mg} \mathrm{kg}^{-1}$ per day sunitinib, and $10 \mathrm{mg} \mathrm{kg}^{-1}$ per day everolimus as a single group (S/E) composed with treatment of alternate drugs administration every week (1W), every 2 weeks (2W), and every 3 weeks (3W). * Symbol stands for statistical analysis of treatment groups relative to control and ${ }^{\S}$ symbol stands for analysis relative to everolimus. ${ }^{\star \star \star}$ or ${ }^{\S \S} P<0.005$, ${ }^{\star \star}$ or ${ }^{\$ \S} P<0.01,{ }^{\star}$ or ${ }^{\S} P<0.05$.

everolimus-based treatments could directly affect angiogenesis (Figure 2C). Among the alternated sequential regimens, the $1 \mathrm{~W}$ regimen displayed the strongest effects on vascular area reduction, whereas the 2 and $3 \mathrm{~W}$ regimens had similar effects as the single agents sunitinib or everolimus.

Discrete effects of sunitinib and everolimus on necrosis and hypoxia induction. Necrosis, as determined by HE staining, was significantly increased in the tumours treated with sunitinib alone or in sunitinib/everolimus sequential combination as compared with vehicle or everolimus alone (Figure 3A). Necrotic areas in tumours treated with sunitinib monotherapy were increased by two-fold reaching $47.3 \%$ of the tumour area compared with $23.6 \%$ in the control-treated tumours (Figure 3B). Similar increase was observed in tumours treated with the alternated sequences of sunitinib and everolimus with necrosis reaching $43.7 \%$ of the tumour area. Taken individually, necrotic areas in the $1 \mathrm{~W}, 2 \mathrm{~W}$, and $3 \mathrm{~W}$ regimens reached $38.1 \%, 52.3 \%$, and $40.6 \%$ of the tumour area, respectively. Treatment with everolimus alone did not induce significant changes in necrotic areas relative to control $(27 \%$ vs $23.6 \%$ ), suggesting that in all regimens, necrosis was a consequence of sunitinib administration.

Staining with anti-CA-IX antibodies was performed to assess occurrence of hypoxia within treated tumours (Figure 3C, Supplementary Figure 4). The CA-IX expression is often regarded as a surrogate marker for HIF expression; the latter being a very unstable biomarker that could be technically difficult to evaluate using immunohistochemistry. Basal CA-IX staining covered 57.7\% of the surface of control tumours (Figure 3D). Sunitinib both as a single agent and in sequential regimens increased the hypoxic area to $69.5 \%$ and $76.1 \%$ of the tumour area, respectively. In the $1 \mathrm{~W}$, $2 \mathrm{~W}$, and $3 \mathrm{~W}$ regimens, hypoxic areas increased to $68.4 \%, 84.1 \%$, and $75.9 \%$ of the tumour area, respectively. In contrast, everolimus alone did not induce significant increase hypoxic areas compared with control (62.7\% vs 57.7\%). As for necrosis, increase in hypoxic areas seemed dependent on sunitinib administration rather than everolimus.

Angiogenesis and mTOR pathway variations according to tumour response. We observed two main tumour behaviours that may characterise the sensitivity to the different treatments: (i) ST tumour control, that is, partial response followed by tumour volume stabilisation, and (ii) tumour PG, that is, tumour volume stabilisation followed by quick disease progression (see Materials and methods). The distribution of mice into the two groups of response was as followed. Forty percent of the tumours were characterised by ST tumour control in the sunitinib $(n=2 / 5)$, everolimus $(n=2 / 5)$, and sunitinib/everolimus treatment groups $(n=6 / 15)$ (see Supplementary Table 1). Slight differences were observed within the alternated regimens, with $40 \%, 20 \%$, and $60 \%$ of ST tumours in the $1 \mathrm{~W}(n=2 / 5), 2 \mathrm{~W}(n=1 / 5)$, and $3 \mathrm{~W}$ $(n=3 / 5)$ regimens, respectively (see Supplementary Table 1). 
A

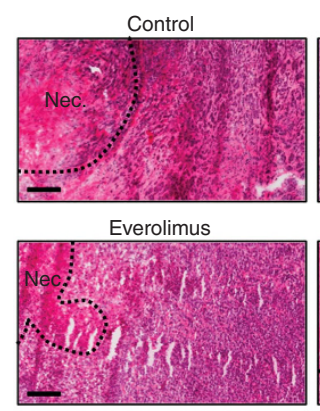

$2 \mathrm{~W}$
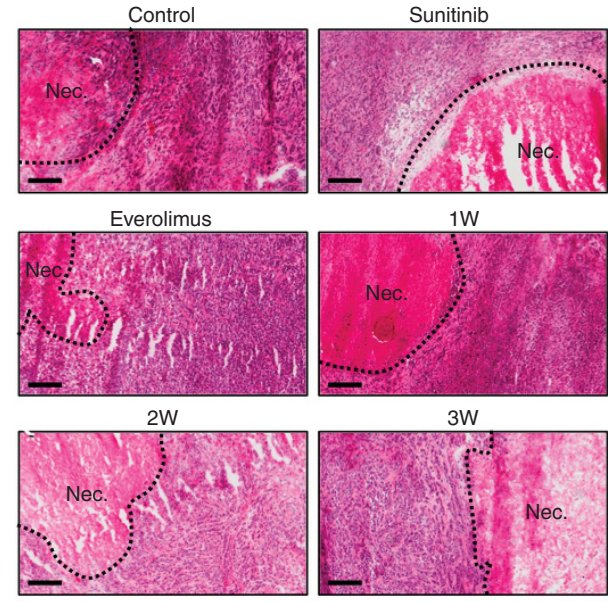

1W

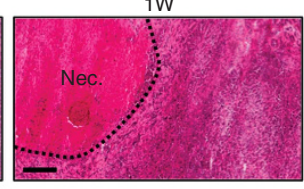

$3 \mathrm{~W}$

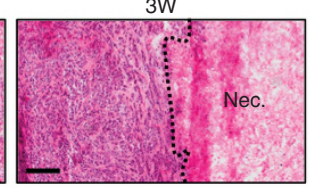

C

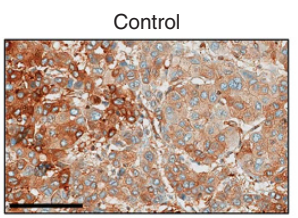

Everolimus

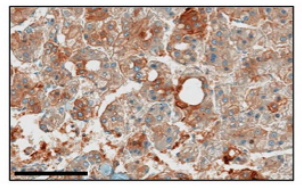

$2 \mathrm{~W}$

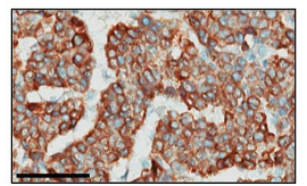

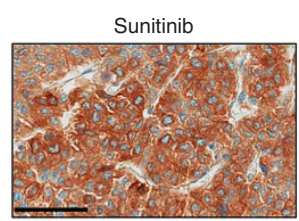

$1 \mathrm{~W}$

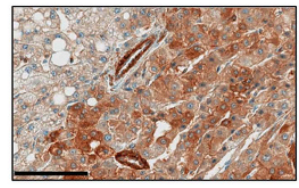

$3 W$

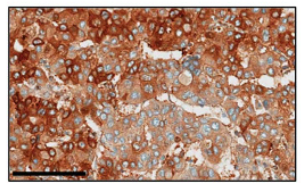

B

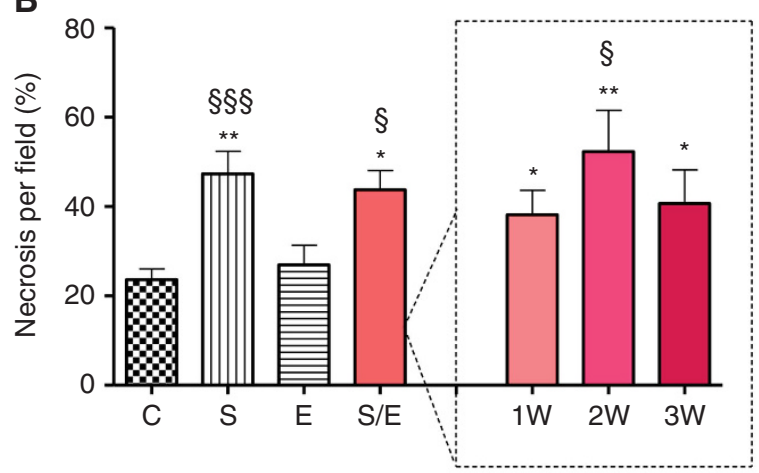

D

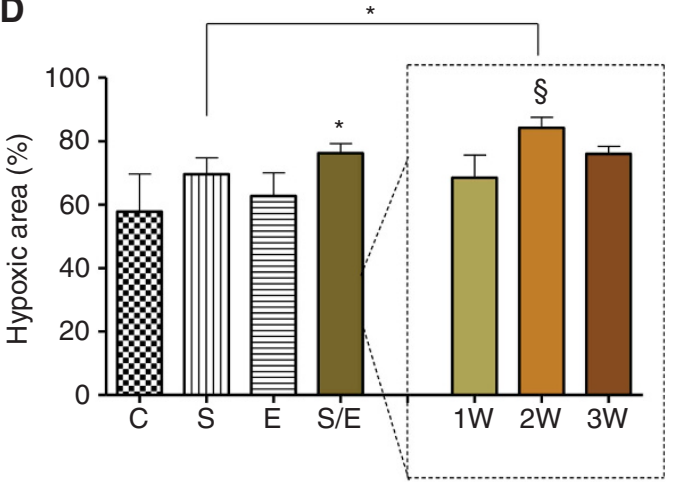

Figure 3. Immunohistochemistry analysis of Caki-1 tumour sections stained with HE and for CA-IX. Representative pictures of HE staining (A) and CA-IX (C) for each group of treatment. Bar graph is $200 \mu \mathrm{m}$ for HE staining and $100 \mu \mathrm{m}$ for CA-IX staining. Necrosis (B) and hypoxia (D) were quantified by Image $\mathrm{J}$ software for mice with tumours treated with vehicle control (C), $60 \mathrm{mg} \mathrm{kg}^{-1}$ per day sunitinib (S), $10 \mathrm{mg} \mathrm{kg}{ }^{-1}$ per day everolimus (E), sequential combination of $60 \mathrm{mg} \mathrm{kg}^{-1}$ per day sunitinib, and $10 \mathrm{mg} \mathrm{kg}^{-1}$ per day everolimus as a single group (S/E) composed with treatment of alternate drugs administration every week (1W), every 2 weeks $(2 W)$, and every 3 weeks ( $3 W$ ). Values represent the means \pm s.e.m. of at least five quantifications per animal with five animals per group, ${ }^{\star}$ Symbol stands for $P$-values relative to control and ${ }^{\S}$ symbol stands for analysis relative to everolimus, ${ }^{\star *}$ or ${ }^{\$} P<0.01,{ }^{*}$ or ${ }^{\S} P<0.05$.

As expression of HIF in our model is not constitutively driven by VHL mutation, changes in tumour angiogenesis and mTOR pathways are considered as potentially adaptive mechanisms in response to the stress induced by sunitinib and everolimus. Vessel numbers and vascular lumen areas were reduced by $2.5-4$-fold in all the treatment groups compared with control, reflecting a strong antiangiogenic effect. This effect was independent of the progression status of the tumours with the exception of tumours controlled by sunitinib alone, which were characterised by reduced vascular lumen area and increased necrosis (Supplementary Figure 2).

We then assessed the expression of the pS6, a downstream target of mTOR (Figure 4A) in all groups of tumours. In controlled tumours, pS6 expression was significantly decreased compared with the pS6 expression in progressive tumours, except for tumours that were treated with sunitinib as a single agent. Everolimus alone, $1 \mathrm{~W}, 2 \mathrm{~W}$, and $3 \mathrm{~W}$ regimens reduced pS6 expression by $60 \%, 38 \%, 70 \%$, and $27 \%$, respectively, in controlled tumours as compared with progressive tumours (Figure 4B). Of note, pS6 expression in progressive tumours was decreased in 2 and $3 \mathrm{~W}$ groups compared with the other regimens (control, 1W, sunitinib, and everolimus monotherapy) indicating a more efficient downregulation of the mTOR pathway with those two sequential combinations. In conclusion, tumours controlled by the treatment were characterised by reduced angiogenesis and mTOR pathway inhibition, whereas progressive-that is, resistant-tumours were characterised by sustained mTOR pathway activity.

Lack of response to targeted therapy and mesenchymal differentiation. Drug-induced hypoxia in tumours may trigger survival pathways among which epithelial-mesenchymal transition (EMT) seems to have a major role. Furthermore, as both angiogenesis and mTOR pathway inhibition may affect cellular differentiation, we evaluated vimentin and E-cadherin expression in tumours (Figure 5A). In the vehicle-treated group, tumours expressed both vimentin $(53.9 \%$ of the tumour area) and E-cadherin (35.7\%) displaying a mixed phenotype with a vimentin/E-cadherin ratio of 1.5 (Figure 5D). In all treatment groups, controlled tumours displayed a decrease in vimentin expression by $73 \%$ and $79 \%$ when treated by sunitinib and everolimus as single agents, respectively, and by $33 \%$ when considering all alternated sequences as a single group. In details, tumour sustainably controlled by $1 \mathrm{~W}, 2 \mathrm{~W}$, and $3 \mathrm{~W}$ regimens were characterised by a reduction in vimentin expression of $42 \%, 46 \%$, and $31 \%$, respectively (Figure 5B). In contrast, E-cadherin expression increased by $93 \%$ in the sunitinib monotherapy group and by $33 \%$ in the alternated sequence groups with some differences among regimens. For instance, controlled tumours in 

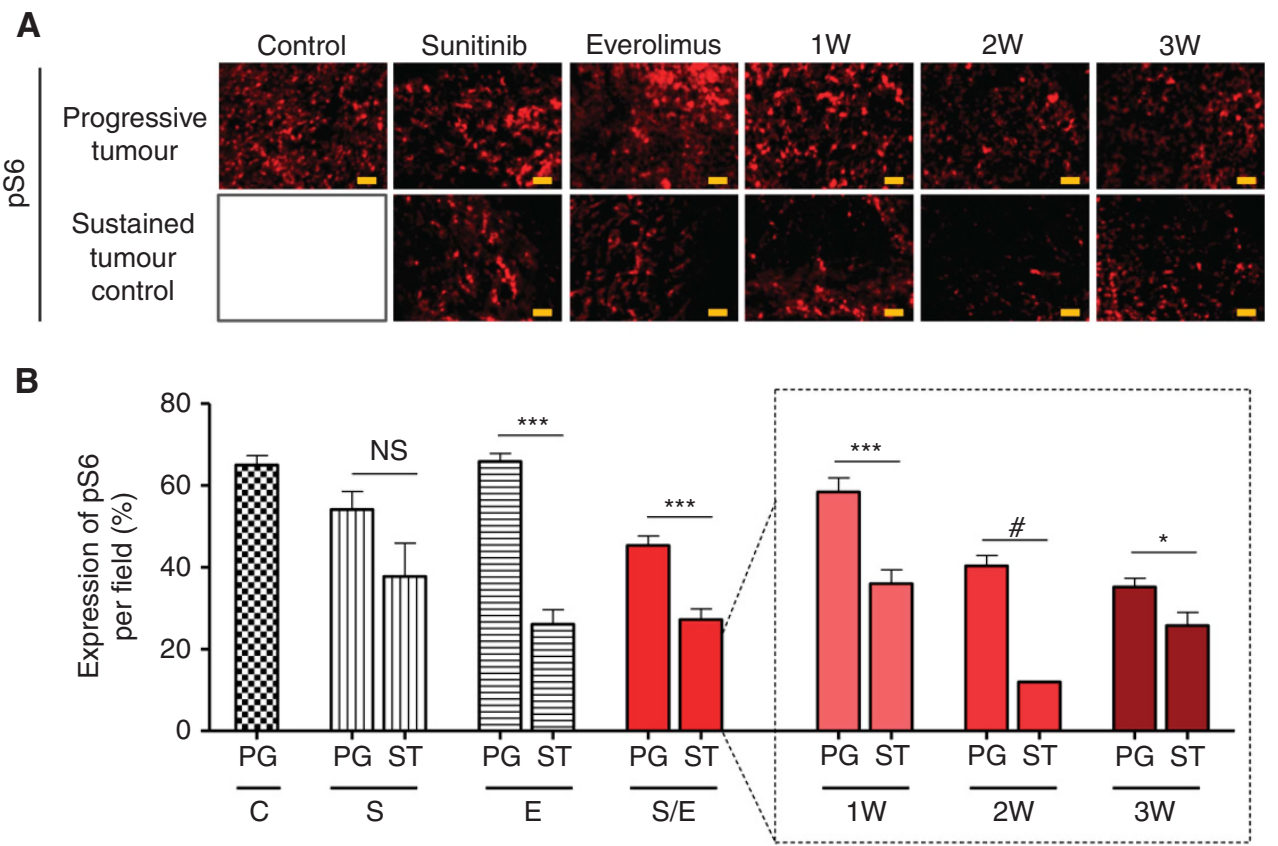

Figure 4. Immunoflorescence analysis of Caki-1 tumour sections stained for pS6. Representative pictures of pS6 staining for each group of treatment (A). Protein expression was quantified by Image $\mathrm{J}$ software for mice with tumours treated with vehicle control (C), $60 \mathrm{mg} \mathrm{kg}{ }^{-1}$ per day sunitinib (S), $10 \mathrm{mg} \mathrm{kg}^{-1}$ per day everolimus (E), sequential combination of $60 \mathrm{mg} \mathrm{kg}^{-1}$ per day sunitinib, and $10 \mathrm{mg} \mathrm{kg}^{-1}$ per day everolimus as a single group (S/E) composed with treatment of alternate drugs administration every week (1W), every 2 weeks (2W), and every 3 weeks (3W). For each treatment group a double bar graph represent in one hand the mice that experienced quick progressive disease (PG) and in the other hand the mice with sustained (ST) tumour control. Values represent the means \pm s.e.m. of at least five quantifications per animal, ${ }^{\star \star \star} P<0.005, \star \star P<0.01$, ${ }^{\star} P<0.05, \mathrm{NS}=$ non significant, ${ }^{*}$ symbol stands for no calculable $P$-values due to the small sample number (B).

the $2 \mathrm{~W}$ and $3 \mathrm{~W}$ groups displayed $70 \%$ and $54 \%$ increase in E-cadherin expression, respectively, whereas tumours treated in the $1 \mathrm{~W}$ regimen displayed a $38 \%$ reduction in E-cadherin expression (Figure 5C). Similar to the $1 \mathrm{~W}$ regimen, controlled tumours in the everolimus monotherapy group displayed a $10 \%$ decrease in E-cadherin expression. Despite these differences among the treatment groups, the ratio of vimentin/E-cadherin expression decreased in all groups compared with the vehicle-treated tumours, suggesting epithelial differentiation when tumours are controlled by the treatments (Figure 5D). Conversely, in most progressive tumours the proportion of vimentin-positive surface did not change, except a slight decrease in the sunitinib monotherapy group (Figure 5B). Three treatment groups displayed reduced E-cadherin expression among progressive tumours compared with control-treated tumours (sunitinib monotherapy, 2 and $3 \mathrm{~W}$ regimens), whereas two treatment groups displayed a modest increase (everolimus monotherapy and $1 \mathrm{~W}$ regimen) (Figure $5 \mathrm{C}$ ). As a consequence, in these progressive tumours the vimentin/ E-cadherin ratio increased in three groups (sunitinib monotherapy, 2 and $3 \mathrm{~W}$ regimens) and slightly decreased in two other groups (everolimus monotherapy and $1 \mathrm{~W}$ regimen) (Figure 5D). These results suggested that progressive tumours under treatment tend to exacerbate a mesenchymal differentiation, especially in the sunitinib monotherapy subgroup, whereas tumours sustainably controlled retain more epithelial phenotypes.

\section{DISCUSSION}

Sunitinib and everolimus are both approved for the treatment of advanced RCC in first-line and second-line therapy, respectively. In most patients, the switch from first-line treatment with TKI to second-line everolimus is made upon occurrence of documented development of tumour PG. Sunitinib is regarded as an antiangiogenic agent inducing vascular disruption, hypoxia, and tumour necrosis. Either as a consequence of pharmacological mechanisms or as a result of constitutively activated hypoxia pathways in the tumour, some clinical data and data from animal models have also suggested that the development of resistance was associated with a more aggressive phenotype. Consequently, attempts have been made to counteract acquired resistance to sunitinib and clinical trials have been developed aiming to switch between active compounds displaying distinct mechanisms of action before the occurrence of acquired resistance.

The aim of our study was to look at changes in human tumours exposed to sequential administration of sunitinib and everolimus to provide information that could serve for clinical trials aiming at delaying tumour PG in RCC patients. In our study, we tested three different sequences of sunitinib and everolimus consisting of every week (1W), every 2 weeks (2W), and every 3 weeks (3W) alternation (Figure 1A). Although the small number of animals limits statistics in terms of TTP in this study, all evaluated sequences yielded longer mTTP than sunitinib and everolimus given as a continuous daily dosing as single agents. This observation supports the hypothesis that treatment alternations before progression could postpone the emergence of tumour PG. In our study, single agent sunitinib yielded shorter mTTP compared with everolimus monotherapy (36 days vs 50 days). Similar observations were made in vivo in other models showing increased median survival of mice treated by everolimus first line compared with sunitinib (Rosa et al, 2013). However, this trend was not observed in clinical trials in which median PFS for firstline sunitinib was consistently superior to everolimus (Motzer et al, 2007, 2013).

Recent data from literature highlighted the possible correlation between EMT and sunitinib resistance in ccRCC (Hammers et al, 2010). Interestingly in a mammary epithelial cells model, the inhibition of mTOR pathway was found to activate EMT (Mikaelian et al, 2013). In our study, tumours treated with everolimus in monotherapy or in alternated sequential regimens 
A
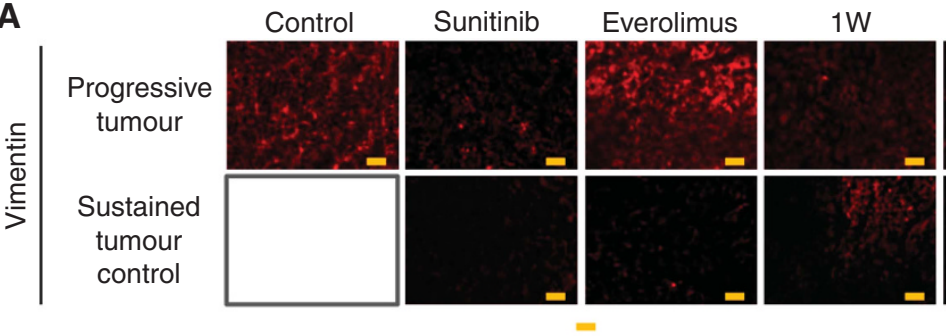

$2 \mathrm{~W}$ $3 \mathrm{~W}$
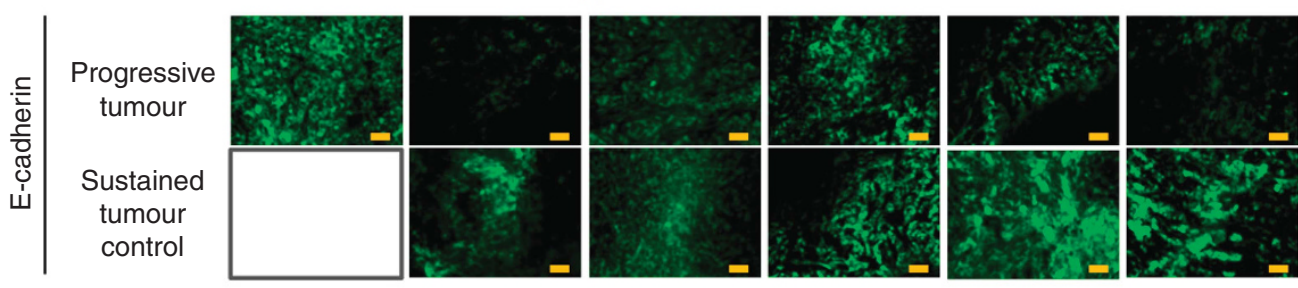

B

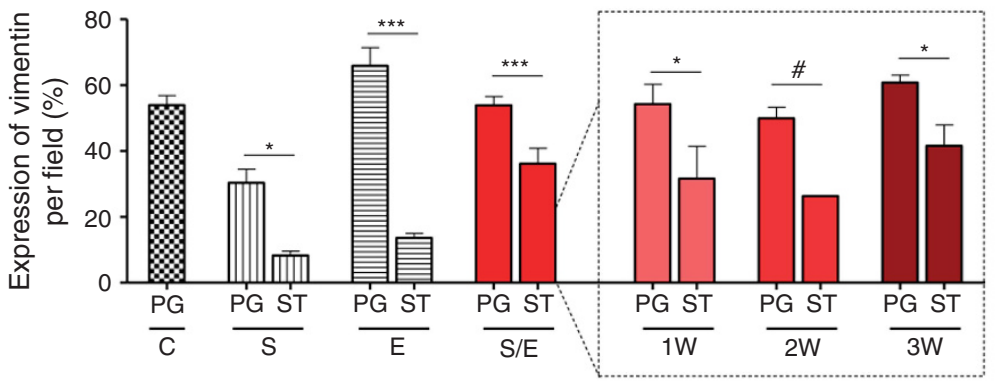

C

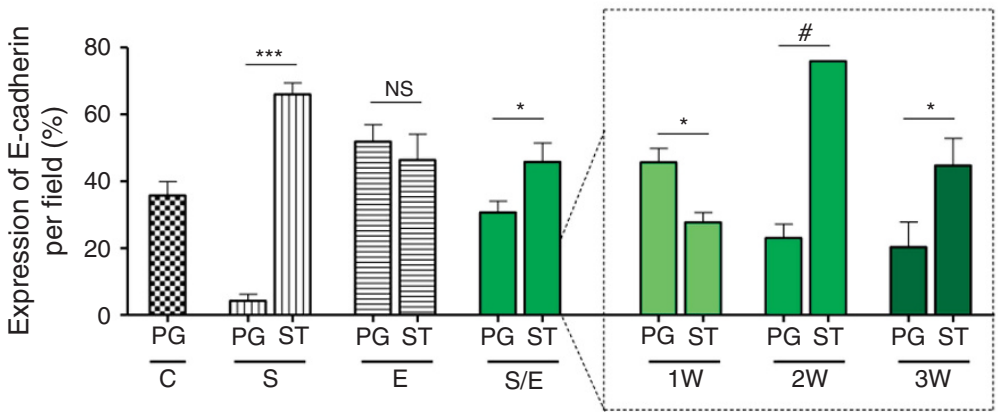

D

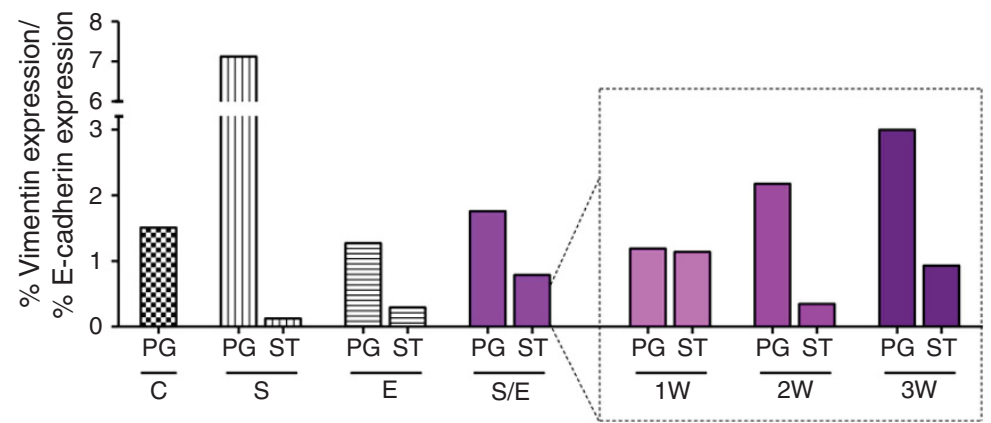

Figure 5. Immunofluorescence analysis of Caki-1 tumour sections stained with EMT markers. Representative pictures of vimentin and E-cadherin staining for each group of treatment (A).Vimentin (B) and E-cadherin (C) expression were quantified by Image $\mathrm{J}$ software for mice with tumours treated with vehicle control (C), $60 \mathrm{mg} \mathrm{kg}^{-1}$ per day sunitinib (S), $10 \mathrm{mg} \mathrm{kg}^{-1}$ per day everolimus (E), sequential combination of $60 \mathrm{mg} \mathrm{kg}^{-1}$ per day sunitinib, and $10 \mathrm{mg} \mathrm{kg}^{-1}$ per day everolimus as a single group (S/E) composed with treatment of alternate drugs administration every week (1W), every 2 weeks $(2 \mathrm{~W})$, and every 3 weeks ( $3 \mathrm{~W})$. Ratios of vimentin expression to E-cadherin expression were calculated in each treatment group (D). For each treatment group a double bar graph represent in one hand the mice with quick progressive disease (PG) and in the other hand the mice with sustained (ST) tumour control. Values represent the means \pm s.e.m. of at least five quantifications per animal with five animals per group, ${ }^{\star \star \star} P<0.005,{ }^{\star \star} P<0.01,{ }^{\star} P<0.05, \mathrm{NS}=$ non significant, ${ }^{*}$ symbol stands for no calculable $P$-values due to the small sample number. 
displayed a higher level of vimentin expression than tumours treated with sunitinib alone consistent with the observation that everolimus may induce mesenchymal differentiation. Interestingly, the ratio of vimentin over E-cadherin expression was different in the alternative sequences compared with monotherapies. This suggests that the tumour cell biology responded differently to alternations of drugs than to continuous exposure to the same inhibitor.

Sequential regimens were all efficient in inhibiting angiogenesis as determined by reduction in the number of tumour vessels and tumour vascular areas. Interestingly, as shown earlier (Lane et al, 2009), angiogenesis inhibition by sunitinib and everolimus proceeded from different mechanisms as neither hypoxia nor necrosis was involved in tumours treated by everolimus alone. In addition, only mice treated with sunitinib monotherapy presented controlled tumours characterised by lower vessel size compared with progressive tumours. In fact, it has been suggested that everolimus was more efficient on mature vessels than sunitinib that mainly affected unstable neoangiogenesis (Lane et al, 2009). Alternate combinations of sunitinib and everolimus may thus target both immature and mature vessels, increasing antiangiogenic activity. New studies are questioning the use of early switch to everolimus after first-line TKI failure instead of a prolonged TKI exposure in second line (Calvani et al, 2013). However, looking at TTP and pS6 inhibition, our data suggested that everolimus remained efficient in a sequential combination strategy in which early switches are done before progression. The degree of pS6 inhibition was even increased in the sequential combination strategy compared with everolimus monotherapy. This may be explained by the fact that programmed switches between everolimus and sunitinib could delay the occurrence of mechanisms associated with drug resistance, such as the induction of AKT activation by a negative feedback loop upon mTOR inhibition that has been previously reported (O'Reilly et al, 2006).

How alternate sequences of sunitinib and everolimus translate into clinical setting remains difficult to evaluate in the absence of specific clinical trial. In the RECORD-3 study comparing sunitinib until progression followed by everolimus $v s$ the reverse sequence, the investigators showed that sunitinib first line followed by everolimus was superior to everolimus first line followed by sunitinib in terms of first-line PFS and overall survival. However, as $>50 \%$ of patients were unable to cross over, it remains difficult to compare second-line with first-line median PFS. However, in the RECORD-1 trial comparing everolimus to placebo after sunitinib or sorafenib failure, median PFS was 4.9 months, which represent $\sim 50 \%$ of first-line median PFS of 10.7 months, assuming a cross trial comparison (Motzer et al, 2007, 2008). In our mice trial, the median TTP was 59 days for the alternated sequences of treatment, which represent a $64 \%$ increased compared with the mTTP of sunitinib. Rosa et al (2013) recently published a study observing the impact of everolimus after 5 weeks of sunitinib treatment and demonstrated that the addition of everolimus slowed down tumour growth compared with sunitinib monotherapy. In their study, they also report a $60 \%$ increase in overall survival for the sequential treatment over monotherapy (97 vs 61 days). All together, these data suggest that a programmed sequential strategy giving sunitinib and everolimus without waiting for progression may be considered as a potential option for designing clinical trials in patients with advanced RCC. In fact, using TKI or everolimus often induce transient toxicities that could be clinically limiting or unbearable for patients leading to therapeutic interruption (Molina et al, 2012). In this study, no significant weight loss or changes in animal behaviour was observed in mice treated with alternate sequences, indicating no toxicity of these treatments (Supplementary Figure 3).

In summary, the use of a sequential combination strategy using alternate treatments with sunitinib and everolimus may be an interesting approach to control tumour PG and postpone the emergence of resistance mechanisms such as the EMT. At least three clinical trials are underway evaluating alternate sequence of sunitinib and everolimus or temsirolimus in patients with advanced RCC (ANZUP 0901, NCT01784978 and NCT01517243).

\section{REFERENCES}

Afonso FJ, Anido U, Fernandez-Calvo O, Vazquez-Estevez S, Leon L, Lazaro M, Ramos M, Anton-Aparicio L (2013) Comprehensive overview of the efficacy and safety of sorafenib in advanced or metastatic renal cell carcinoma after a first tyrosine kinase inhibitor. Clin Transl Oncol 15: 499.

Calvani N, Morelli F, Chiuri V, Gnoni A, Scavelli C, Fedele P, Orlando L, Maiello E, Lorusso V, Cinieri S (2013) Prolonged exposure to tyrosine kinase inhibitors or early use of everolimus in metastatic renal cell carcinoma: are the two options alike? Med Oncol 30: 578.

Chiatar SS, Eze OP, Schoenfeld AR (2013) Expression of VHL causes three-dimensional morphological changes in renal cells indicative of proximal tubule differentiation. Cell Dev Biol 2: e-pub ahead of print 12 August 2013; doi:10.4172/2168/9296.1000118.

Cockman ME, Masson N, Mole DR, Jaakkola P, Chang GW, Clifford SC, Maher ER, Pugh CW, Ratcliffe PJ, Maxwell PH (2000) Hypoxia inducible factor-alpha binding and ubiquitylation by the von Hippel-Lindau tumor suppressor protein. J Biol Chem 275: 25733-25741.

Cohen HT, McGovern FJ (2005) Renal-cell carcinoma. N Engl J Med 353: 2477-2490.

Creighton CJ, Morgan M, Gunaratne PH, Wheeler DA, Gibbs RA, Robertson A, Chu A, Beroukhim R, Cibulskis K (2013) Comprehensive molecular characterization of clear cell renal cell carcinoma. Nature 499 . 43-49.

Ebos JM, Lee CR, Cruz-Munoz W, Bjarnason GA, Christensen JG, Kerbel RS (2009) Accelerated metastasis after short-term treatment with a potent inhibitor of tumor angiogenesis. Cancer Cell 15: 232-239.

Faivre S, Delbaldo C, Vera K, Robert C, Lozahic S, Lassau N, Bello C, Deprimo S, Brega N, Massimini G, Armand JP, Scigalla P, Raymond E (2006a) Safety, pharmacokinetic, and antitumor activity of SU11248, a novel oral multitarget tyrosine kinase inhibitor, in patients with cancer. J Clin Oncol 24: 25-35.

Faivre S, Demetri G, Sargent W, Raymond E (2007) Molecular basis for sunitinib efficacy and future clinical development. Nat Rev Drug Discov 6: 734-745.

Faivre S, Kroemer G, Raymond E (2006b) Current development of mTOR inhibitors as anticancer agents. Nat Rev Drug Discov 5: 671-688.

Fuereder T, Jaeger-Lansky A, Hoeflmayer D, Preusser M, Strommer S, Cejka D, Koehrer S, Crevenna R, Wacheck V (2010) mTOR inhibition by everolimus counteracts VEGF induction by sunitinib and improves antitumor activity against gastric cancer in vivo. Cancer Lett 296: 249-256.

Gassenmaier M, Chen D, Buchner A, Henkel L, Schiemann M, Mack B, Schendel DJ, Zimmermann W, Pohla H (2013) CXC chemokine receptor 4 is essential for maintenance of renal cell carcinoma-initiating cells and predicts metastasis. Stem Cells 31: 1467-1476.

Gossage L, Murtaza M, Slatter AF, Lichtenstein CP, Warren A, Haynes B, Marass F, Roberts I, Shanahan SJ, Claas A, Dunham A, May AP, Rosenfeld N, Forshew T, Eisen T (2014) Clinical and pathological impact of VHL, PBRM1, BAP1, SETD2, KDM6A, and JARID1c in clear cell renal cell carcinoma. Genes Chromosomes Cancer 53: 38-51.

Hammers HJ, Verheul HM, Salumbides B, Sharma R, Rudek M, Jaspers J, Shah P, Ellis L, Shen L, Paesante S, Dykema K, Furge K, Teh BT, Netto G, Pili R (2010) Reversible epithelial to mesenchymal transition and acquired resistance to sunitinib in patients with renal cell carcinoma: evidence from a xenograft study. Mol Cancer Ther 9: 1525-1535.

Huang B, Huang YJ, Yao ZJ, Chen X, Guo SJ, Mao XP, Wang DH, Chen JX, Qiu SP (2013) Cancer stem cell-like side population cells in clear cell renal cell carcinoma cell line 769 P. PLoS One 8: e68293.

Keisner SV, Shah SR (2011) Pazopanib: the newest tyrosine kinase inhibitor for the treatment of advanced or metastatic renal cell carcinoma. Drugs 71: 443-454.

Lane HA, Wood JM, McSheehy PM, Allegrini PR, Boulay A, Brueggen J, Littlewood-Evans A, Maira SM, Martiny-Baron G, Schnell CR, Sini P, O’Reilly T (2009) mTOR inhibitor RAD001 (everolimus) has antiangiogenic/vascular properties distinct from a VEGFR tyrosine kinase inhibitor. Clin Cancer Res 15: 1612-1622. 
Larkin J, Esser N, Calvo E, Tsuchihashi Z, Fiedler U, Graeser R, Kim D (2012) Efficacy of sequential treatment with sunitinib-everolimus in an orthotopic mouse model of renal cell carcinoma. Anticancer Res 32: 2399-2406.

Ljungberg B, Bensalah K, Bex A, Canfield S, Dabestani S, Hofmann F, Hora M, Kuczyk MA, Lam T, Marconi L, Merseburger AS, Mulders PFA, Staehler M, Volpe A (2013) EAU guidelines on renal cell carcinoma: the 2013 update. European assosiation of Urology. http://www.uroweb.org/gls/ pdf/10_Renal_Cell_Carcinoma_LR.pdf.

Lu J, Cui Y, Zhu J, He J, Zhou G, Yue Z (2013) Biological characteristics of Rh123 stem-like cells in a side population of 786-O renal carcinoma cells. Oncol Lett 5: 1903-1908.

Makhov PB, Golovine K, Kutikov A, Teper E, Canter DJ, Simhan J, Uzzo RG, Kolenko VM (2012) Modulation of Akt/mTOR signaling overcomes sunitinib resistance in renal and prostate cancer cells. Mol Cancer Ther 11: 1510-1517.

Mikaelian I, Malek M, Gadet R, Viallet J, Garcia A, Girard-Gagnepain A, Hesling C, Gillet G, Gonzalo P, Rimokh R, Billaud M (2013) Genetic and pharmacologic inhibition of mTORC1 promotes EMT by a TGF-betaindependent mechanism. Cancer Res 73: 6621-6631.

Molina AM, Feldman DR, Voss MH, Ginsberg MS, Baum MS, Brocks DR, Fischer PM, Trinos MJ, Patil S, Motzer RJ (2012) Phase 1 trial of everolimus plus sunitinib in patients with metastatic renal cell carcinoma. Cancer 118: 1868-1876.

Motzer RJ, Barrios CH, Kim TM, Falcon S, Cosgriff T, Harker WG, Pittman KB, Sabbatini R, Rha SY, Flaig TW, Page RD, Bavbek SE, Beck JT, Patel PM, Schiff E, Vaury A, Niolat J, Gogov S, Anak O, Knox J (2013) Record-3: Phase II randomized trial comparing sequential first-line everolimus (EVE) and second-line sunitinib (SUN) versus first-line SUN and second-line EVE in patients with metastatic renal cell carcinoma (mRCC). In 2013 ASCO Annual meeting J Clin Oncol 31: 2765-2772.

Motzer RJ, Escudier B, Oudard S, Hutson TE, Porta C, Bracarda S, Grunwald V, Thompson JA, Figlin RA, Hollaender N, Kay A, Ravaud A (2010) Phase 3 trial of everolimus for metastatic renal cell carcinoma: final results and analysis of prognostic factors. Cancer 116: 4256-4265.

Motzer RJ, Escudier B, Oudard S, Hutson TE, Porta C, Bracarda S, Grunwald V, Thompson JA, Figlin RA, Hollaender N, Urbanowitz G,
Berg WJ, Kay A, Lebwohl D, Ravaud A (2008) Efficacy of everolimus in advanced renal cell carcinoma: a double-blind, randomised, placebocontrolled phase III trial. Lancet 372: 449-456.

Motzer RJ, Hutson TE, Tomczak P, Michaelson MD, Bukowski RM, Rixe O, Oudard S, Negrier S, Szczylik C, Kim ST, Chen I, Bycott PW, Baum CM, Figlin RA (2007) Sunitinib versus interferon alfa in metastatic renal-cell carcinoma. $N$ Engl J Med 356: 115-124.

O’Reilly KE, Rojo F, She QB, Solit D, Mills GB, Smith D, Lane H, Hofmann F, Hicklin DJ, Ludwig DL, Baselga J, Rosen N (2006) mTOR inhibition induces upstream receptor tyrosine kinase signaling and activates Akt. Cancer Res 66: 1500-1508.

Paez-Ribes M, Allen E, Hudock J, Takeda T, Okuyama H, Vinals F, Inoue M, Bergers G, Hanahan D, Casanovas O (2009) Antiangiogenic therapy elicits malignant progression of tumors to increased local invasion and distant metastasis. Cancer Cell 15: 220-231.

Pugh CW, Ratcliffe PJ (2003) Regulation of angiogenesis by hypoxia: role of the HIF system. Nat Med 9: 677-684.

Rosa R, Damiano V, Nappi L, Formisano L, Massari F, Scarpa A, Martignoni G, Bianco R, Tortora G (2013) Angiogenic and signalling proteins correlate with sensitivity to sequential treatment in renal cell cancer. Br J Cancer 109: 686-693.

Schoffski P, Dumez H, Clement P, Hoeben A, Prenen H, Wolter P, Joniau S, Roskams T, Van Poppel H (2006) Emerging role of tyrosine kinase inhibitors in the treatment of advanced renal cell cancer: a review. Ann Oncol 17: 1185-1196.

Semenza GL (2013) HIF-1 mediates metabolic responses to intratumoral hypoxia and oncogenic mutations. J Clin Invest 123: 3664-3671.

Zhang Y, Sun B, Zhao X, Liu Z, Wang X, Yao X, Dong X, Chi J (2013) Clinical significances and prognostic value of cancer stem-like cells markers and vasculogenic mimicry in renal cell carcinoma. J Surg Oncol 108: 414-419.

This work is published under the standard license to publish agreement. After 12 months the work will become freely available and the license terms will switch to a Creative Commons AttributionNonCommercial-Share Alike 3.0 Unported License.

Supplementary Information accompanies this paper on British Journal of Cancer website (http://www.nature.com/bjc) 